\title{
The Effect of Local Endometrial Injury and GnRH Agonist on Pregnancy Rate in Patients With Recurrent Implantation Failure
}

\author{
Laeia Farzadi $^{1}$, Arefeh Fakour ${ }^{2}$, Alieh Ghasemzadeh ${ }^{1}$, Kobra Hamdi $^{1}$, Sedigheh Abdollahi Fard ${ }^{1}$, \\ Mohammad Nouri ${ }^{1}$, Behroz Niknafs ${ }^{1}$, Vahideh Shahnazi ${ }^{1}$
}

\begin{abstract}
Objectives: These days, infertility is one of the most common diseases in the community involving $10 \%-15 \%$ of the couples. One method of infertility treatment is assisted reproductive technology (ART). Recently studies have shown that local endometrial injury in patients treated with in vitro fertilization (IVF) cause treatment failure and subsequently decrease the implantation rates. The aim of this study was to show the direct relationship between local endometrial injury and increasing recurrent implantation failure (RIF).

Materials and Methods: Our study populations included patients that experienced at least two or more RIFs during IVF which was performed in the Department of Obstetrics and Gynecology of Tabriz University of Medical Sciences.

Results: In this study, 76 patients that experienced at least two or more RIFs during IVF were studied. Any local endometrial injury was examined in regard to its affect on the pregnancy rate. The results showed that there is not any relationship between the mean duration of infertility with and without endometrial trauma and IVF in patients.

Conclusion: Seven patients with endometrial trauma and 6 patients without endometrial trauma before IVF showed positive $\beta$ HCG test. Meanwhile there were not significant differences between $\beta$ HCG test in patients with and without local endometrial injury before IVF. Significant correlation was not found between the incidences of pregnancy after IVF in patients with other parameters. Keywords: Endometrial, Fertilization in vitro, Implantation, Trauma
\end{abstract}

\section{Introduction}

Infertility is defined as the failure of pregnancy following one year of unprotected sexual intercourse without using contraception. Approximately $85 \%-90 \%$ of healthy couples will be pregnant within 6 months in a year, and often infertility affects about $10 \%-15 \%$ of couples (1).

Contrary to public perception, the fertility rate has remained relatively unchanged over the past 3 decades, however, the evaluation and treatment of infertility has changed dramatically over the same period. Several important advances in this field have the greatest impact; the most important of which are in vitro fertilization (IVF) and assisted reproductive technology (ART) (1).

Approximately one third of women who have attempted for IVF have become pregnant. Thus, failure in this process implies on a defect in implantation of embryo or shortly after it (1). Although delivery rate has improved enormously following embryo transfer during IVF, it is clear that the majority of IVF-ET cycles are still not successful, and in most cases, an obvious explanation does not exist except for the failure of the replacement process (1). Controlled ovarian hyper $(\mathrm{COH})$ stimulation is an im- portant factor in the success of IVF-ET. However, despite great advances in ovarian stimulation protocols and fertilization, implantation per embryos is roughly $15 \%$, thus a lot of couples have left fertility treatment after failure in pregnancy (2).

In couples with recurrent implantation failure (RIF) (i.e. after 3 cycles of good embryo transfer are not pregnant) a series of multiple therapeutic strategies have been proposed, including hysteroscopy, endometrial trauma and changes in stimulation protocols, blastocyst assisted hatching transfer and pre-implantation genetic diagnosis (PGD) for aneuploidy. The advantage of the strategies over each other, however, is not clear.

Local trauma to the endometrium in candidate patients for IVF may lead to acidolization and increased potential of implantation failure (2).

Prognostic factors in IVF success depends on factors including maternal age, ovarian reserve status and pre-ability of the reproductive system. Younger women with normal ovarian reserve have more probability of pregnancy in comparison with older woman with diminished ovarian reserve. Also, women who have previously failed IVF 
have less success rate of pregnancy. The prognosis of IVF in women with endometriosis is poorer than those with other risk factors.

Today, considering the changes in the demographic characteristics of the community which has led the women to become pregnant at an older age and also from the biological perspective in these women, child bearing potential has become less. The significance of ART and related measures are identified more in cases of failure of implantation in 2 or more than 2 times (1).

Thus, the present study showed the improved outcomes of IVF in patients with previous IVF failure. It seems that measures taken for the patients in this study increase implantation compensation and clinical pregnancy. In this investigation, in ART candidates who have been nominated in two or more implant failures, implantation rates are increased using oral contraceptive (OCP) and local uterine damage through papilla biopsy method on 19th day of menstrual cycle and use of induction of ovulation with triptorelin which is a short-acting GnRH agonist. Follow-up was done with ultrasound and estradiol.

Improvement of acceptance of endometrium is essential to improving the success rate of implantation. Medical and surgical treatment of the basic gynecological diseases improves uterine reception. Manipulation of gene expression of endometrial tissue with the help of genetic science and fundamental methods may lead to further improvement in implantation rates, someday (2).

In a study, Cakmak and Taylor from Yale University of America pointed out that suppression or lack of essential molecules for acceptance of uterine prompts decrease implantation in animal models as well as human suffering from gynecological diseases such as endometriosis, hydrosalpinx, leiomyoma and PCO; however the mechanism of failure of implantation is not yet fully determined but it seems to be caused by cytokine disorder and abnormal impaired hormonal signals such as epigenetic alterations (2).

In the study by Orvieto et al on 19 patients with a history of multiple failed IVF, patients utilized a combination of diagnostic hysteroscopy and endometrial biopsy (uterine localized damage) associated with oral contraceptive tablet and short-acting GnRH analog protocol data was applied after discontinuation. Patients, who were supervised for a combined approach of treatment, achieved improved implantation rates and clinical pregnancy (25\% and $42 \%$ in the implantation rates and clinical pregnancy, respectively) (3).

The study of Karimzadeh et al from the Yazd University of Medical Sciences showed that implantation failure is the leading cause of failure in IVF or intracytoplasmic sperm injection (ICSI). It is reported that localized endometrial damage caused by biopsy catheter will result in increased pregnancy rates in subsequent cycles of treatment. Local endometrial injury, through release of mediators such as histamine and growth factors, increases implantation rates. The rate of implantation in this study was $10.9 \%$ in localized endometrial damage group against 3.3\% in control group. Pregnancy rate in endometrial biopsy or local injuries had an amount of $27 \%$ rather than $8.9 \%$ in control group (4).

Zhou et al of Peking University in China studied 60 patients among 121 women undergoing $\mathrm{COH}$. From the study it is concluded that these measures result in improved embryo implantation rates and raises clinical pregnancy newborns (33\% in endometrial implantation trauma group and $17 \%$ in control group) (5).

The aim of this study is to show the relationship among local endometrial injury, pregnancy rate and RIF.

\section{Materials and Methods}

In a clinical trial conducted on patients who experienced at least 2 or more RIF in IVF in Department of Obstetrics and Gynecology of Tabriz University of Medical Sciences, the rate of pregnancy in women with endometrial trauma who experienced RIF was investigated.

According to the results of previous studies the success rate of pregnancy in normal group with routine IVF, patients with RIF (Repeated Implantation Failure) and those with endometrial trauma was reported as 33\%, $10 \%$ and $17 \%$, respectively.

For this study, 76 cases that were randomly divided into two groups were selected. The inclusion criteria included patients that had two or more implantation failures in IVF. Exclusion criteria included no increase follicle size over 17-18 mm, no increase in estradiol levels over 150$400 \mathrm{pg}$, thin endometrium and patient dissatisfaction. In the Infertility Department of Al Zahra hospital, random sampling was conducted on patients with two or more implantation failures. Considering that our target group consisted of patients with the RIF (twice or more) in IVF, who were candidates for ovulation stimulation, ovarian puncture and IVF, thus the cases were divided into two groups. On the 19th day of the menstrual cycle, endometrial trauma was applied in the first group while the control group (second one) had no trauma.

In both groups, ovulation stimulation was performed by use of ampoule Superfast $500 \mu \mathrm{g} / \mathrm{sc}$ (GnRH agonist), with the routine IVF being the same in both. After embryo transfer (Fresh - ET), endometrial receptivity and pregnancy rate were compared between two groups.

From the fifth day of the cycle, contraceptive pills (LD) were prescribed for 21 days and the pipelle biopsy was performed on the 19th day of the menstrual cycle. From 21st day of the cycle, a subcutaneous injection of the superfast $(500 \mu \mathrm{g})$ was prescribed. On the first and second days of the menstrual cycle, the patients returned to the hospital. After performing transvaginal ultrasonography-whereas endometrium appeared normal and there were no ovarian cysts-ovulation induction with exogenous gonadotropins (preferably Gonal-F or monogon) was conducted and follicular growth monitoring by transvaginal ultrasonography and measuring the level of estradiol serum were subsequently performed. While there were appropriate 
follicles (3-4 follicles with a diameter of 17-18 mm) and suitable serum estradiol level (150-400 pg/ml), superfast was stopped and one dose (10000 IU) of intramuscular human chorionic gonadotropin (HCG) injection was prescribed and 24-36 hours later the follicular puncture was started. Finally after the formation of the embryos, 48 hours after oocyte retrieval, the embryo transferring was began. Luteal phase was supported by progesterone.

\section{Statistical Analysis}

Data were entered into statistical software Spss 17.0. The statistical test, chi-square, Man-Whitney $\mathrm{U}$, and $t$ test was used for statistical analysis with $P<0.005$ was considered statistically significant.

\section{Results}

The present study was conducted on 76 IVF patients with two implantation failures. In this study, the effect of endometrial injury on the rate of pregnancy of patients with history of RIF was studied and the following results were obtained.

The average age of women with endometrial trauma was 33.444 .32 years while the average age of women without endometrial trauma was $32.26 \pm 4.91$ years $(P=0.269)$.

The average duration of infertility for women with endometrial trauma and without endometrial trauma was 9.72 \pm 4.04 years and $8.58 \pm 5.37$ years, respectively $(P=0.312)$. Results of the $\beta \mathrm{HCG}$ test performed in 7 patient with endometrial trauma and 6 patients without endometrial trauma were positive $(P=0.761)$. The average uses of ART in women with endometrial trauma and women without endometrial trauma were $3.68 \pm 1.21$ and $3.26 \pm 0.94$, respectively $(P=0.096)$.

The average level of estradiol in women with endometrial trauma and women without endometrial trauma was $1773.42 \pm 675.03$ and $1845.26 \pm 769.77 \mathrm{pg} / \mathrm{ml}$, respectively $(P=0.667)$. The average thickness of the endometrium in women with endometrial trauma and women without endometrial trauma was $9.18 \pm 1.22 \mathrm{~mm}$ and $8.981 .09 \mathrm{~mm}$, respectively $(P=0.461)$. The average number of mature follicles was $8.92 \pm 3.94$ and $8.76 \pm 3.68$ in women with endometrial trauma and women without endometrial trauma, respectively $(P=0.857)$. A total of 29 patients with endometrial trauma and 27 without endometrial trauma suffered from primary infertility.

\section{Discussion}

With the increasing advancement of human knowledge, major advancements have been achieved in the treatment of infertility. One of the solutions to this problem is the ART.

In a study carried out by Barash et al on IVF patients, the effect of local endometrial local trauma was analyzed. These researches reported that the rate of pre-IVF successful implantation (pregnancy) in women with endometrial local trauma was $27.7 \%$ and in women without endometrial trauma was $14.2 \%$. The rate of success was significantly higher in women with endometrial local trauma prior to IVF. Moreover, the rate of pregnancy was also higher in the latter group (6).

In the present study, the rate of pre-IVF pregnancy in women with endometrial trauma was $18.4 \%$, which was higher than the rate of pregnancy in women without endometrial trauma (15.7\%). Similar to the results of the aforementioned research, the rate of pre-IVF pregnancy was higher in women with endometrial trauma.

Karimzadeh et al. of the Shahid Sadouqi University of Yazd conducted a study on patients with repeated failed implantation. They analyzed the effect of local endometrial trauma on pre-IVF patients and found out that IVF local trauma leads to a significant increase in the success of implantation in patients with history of repeated unsuccessful implantation. That is to say, the rate of successful implantation in patients with endometrial trauma was $10.9 \%$ while the rate of success in the control group was $3.38 \%$ (4).

In another research performed by Li et al in the Infertility Department of Shenzhen University of China it was found out that local endometrial trauma leads to an increase in the success of implantation in IVF patients (7).

In a study by Zhou et al in the Infertility Department of Shenzhen University of China, a number of IVF patients were examined. Results of this research revealed that preIVF local endometrial trauma leads to an increase in the success of IVF in patients (8).

Baum et al carried out a study to analyze the effect of endometrial local trauma on the rate of successful implantation in IVF patients. They reported that pre-IVF local trauma does not leave a significant effect on successful implantation in such patients but improves it (9).

In 2012, El-Toukhy et al carried out a research in the Thomas Hospital (London) to study the effect of local endometrial trauma on IVF patients. They stated that according to the studies on the effect of local trauma as well as the effect of this method on the improvement of IVF implantation success, endometrial local trauma leads to an increase in the rate of successful implantation in clinical trials (10).

In another study by Potdar et al in the Leicester University (England) the effect of endometrial trauma on IVF patients was examined. These researchers reported that this method improves successful implantation in $70 \%$ of patients (11).

Shohayeb et al carried out a study in the IVF center Department of Obstetrics and Gynecology of Cario University in 2012. They studied IVF patients and reported that pre-IVF endometrial biopsy leads to a significant increase in the successful implantation in these patients. That is to say, the success of implantation in biopsied patients was $12 \%$ while the rate of success in the control group was $7 \%$. Moreover, clinical pregnancy observed in biopsied patients prior to IVF was $32 \%$, while the clinical pregnancy in the control group was $18 \%(12)$.

In the present study, although the rate of pre-IVF preg- 
nancy in patient with endometrial trauma was higher than that of patients without endometrial trauma, no significant difference was observed between the pregnancy rates of these two groups.

\section{Conclusion}

In this study, a total of 76 IVF patients with at least two failed implantations were studied and analyzed about the effect endometrial trauma on pregnancy rate of patients whom experienced RIF. However, no significant difference was observed between the average duration of infertility in patient with and without pre-IVF endometrial trauma. Results of the $\beta$ HCG test in 7 patients with endometrial trauma prior to IVF and 6 patients without endometrial trauma prior to IVF were positive.

There were not significant differences between $\beta$ HCG test in patients with and without local endometrial injury before IVF. Furthermore, there were not significant relationship among the rates of post-IVF pregnancy and other parameters in the participants.

\section{Ethical issues}

For all patients who entered the study, written informed consent was obtained after giving the necessary information and the effects and consequences of the acts described to patients in an understandable language. This clinical trial is registered at http://www.irct.ir/ as IRCT2013072412146N2.

\section{Conflict of interests}

The authors declare no conflict of interests.

\section{Financial support}

This study was supported by Research Deputy of Tabriz University of Medical Sciences.

\section{Acknowledgments}

We would like to thank Womans Reproductive Health Research Center, Tabriz University of Medical Sciences, Iran.

\section{References}

1. Speroff L, Fritz MA. Clinical Gynecologic Endocrinology and Infertility. 7th edition. Philadelphia: Lippincott Williams \& Wilkins; 2010:1013-1068.

2. Cakmak H, Taylor HS. Implantation failure: molecular mechanisms and clinical treatment. Hum Reprod Update. 2011;17(2):242-253. doi:10.1093/ humupd/dmq037.
3. Orvieto R, Meltcer S, Liberty G, Rabinson J, Anteby EY, Nahum R. A combined approach to patients with repeatedIVFfailures. FertilSteril.2010;94(6):24622464. doi:10.1016/j.fertnstert.2010.03.057.

4. Karimzadeh MA, Ayazi Rozbahani M, Tabibnejad N. Endometrial local injury improves the pregnancy rate among recurrent implantation failure patients undergoing in vitro fertilisation/intra cytoplasmic sperm injection: a randomised clinical trial. Aust N Z J Obstet Gynaecol. 2009;49(6):677-680. doi: 10.1111/j.1479-828X.2009.01076.x.

5. Zhou L, Li R, Wang R, Huang HX, Zhong K. Local injury to the endometrium in controlled ovarian hyperstimulation cycles improves implantation rates. Fertil Steril. 2008;89(5):1166-1176.

6. Barash A, Dekel N, Fieldust S, Segal I, Schechtman E, Granot I. Local injury to the endometrium doubles the incidence of successful pregnancies in patients undergoing in vitro fertilization. Fertil Steril. 2003;79(6):1317-1322.

7. Li R, Hao G. Local injury to the endometrium: its effect on implantation. Curr Opin Obstet Gynecol. 2009;21(3):236-9. doi:10.1097/GCO.0b013e32832a0654.

8. Zhou L, Li R, Wang R, Huang HX, Zhong K. Local injury to the endometrium in controlled ovarian hyperstimulation cycles improves implantation rates. Fertil Steril. 2008;89(5):1166-1176.

9. Baum M, Yerushalmi GM, Maman E, et al. Does local injury to the endometrium before IVF cycle really affect treatment outcome? Results of a randomized placebo controlled trial. Gynecol Endocrinol. 2012;28(12):933-936. doi:10.3109/09513590.2011.65 0750 .

10. El-Toukhy T, Sunkara SK, Coomarasamy A, Grace J, Khalaf Y. Outpatient hysteroscopy and subsequent IVF cycle outcome: a systematic review and metaanalysis. Reprod Biomed Online. 2008;16(5):712-719.

11. Potdar N, Gelbaya T, Nardo LG. Endometrial injury to overcome recurrent embryo implantation failure: a systematic review and meta-analysis. Reprod Biomed Online. 2012;25(6):561-571. doi:10.1016/j. rbmo.2012.08.005.

12. Shohayeb A, El-Khayat W. Does a single endometrial biopsy regimen (S-EBR) improve ICSI outcome in patients with repeated implantation failure? A randomised controlled trial. Eur J Obstet Gynecol Reprod Biol. 2012;164(2):176-179. doi:10.1016/j. ejogrb.2012.06.029.

Copyright (c) 2016 The Author(s); This is an open-access article distributed under the terms of the Creative Commons Attribution License (http://creativecommons.org/licenses/by/4.0), which permits unrestricted use, distribution, and reproduction in any medium, provided the original work is properly cited. 